\title{
Clinical and prognostic significance of pat
and inflammatory markers in the surgical treatment of locally advanced colorectal cancer
}

This article was published in the following Dove Press journal:

OncoTargets and Therapy

I September 2015

Number of times this article has been viewed

\section{Sokolov' \\ K Angelov' \\ MVasileva' \\ MP Atanasova ${ }^{2}$}

A Vlahova ${ }^{3}$

G Todorov'

'Department of Surgery, ${ }^{2}$ Department of Anesthesiology and Intensive Care, ${ }^{3}$ Department of Pathology, University Hospital Alexandrovska, Medical University of Sofia, Sofia, Bulgaria

Video abstract

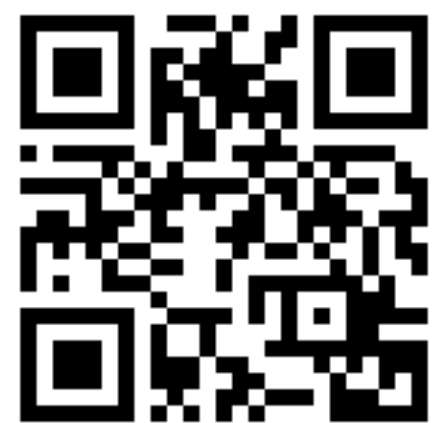

Point your SmartPhone at the code above. If you have a QR code reader the video abstract will appear. Or use: http://youtu.be/es5csllbVdc

Correspondence: Manol Sokolov Department of Surgery, University Hospital Alexandrovska, Medical University of Sofia, Georgi Sofiyski str I, Sofia 1431, Bulgaria

Tel +35929230540

Fax +35929230345

Email m69sokolov@abv.bg
Background: Locally advanced colorectal cancer (CRC) may vary in its clinical and pathological appearance. It is now accepted that progression of disease in patients with locally advanced CRC is determined not only by local tumor characteristics but also by the immune system and inflammatory response in the body.

Methods: We investigated patients with confirmed CRC who were treated in the surgical clinic at the University Hospital Alexandrovska over a 10-year period and retrospectively evaluated the histological features of the preoperative biopsies and operative specimens removed during radical multivisceral resections. We also collected prospective data for serum C-reactive protein levels and Jass-Klintrup score, Petersen Index score, and Glasgow Prognostic Score in patients with locally advanced CRC.

Results: Of 1,105 patients with CRC, 327 (29.6\%) were diagnosed with locally advanced disease. In total, 108 combined multivisceral resections (79 for primary tumors and 29 for recurrent tumors) were performed. Overall survival was 34 months for $\mathrm{pR} 0$ cases and 12 months for $\mathrm{pR} 1$ cases $(P<0.05)$. Our data confirmed that $\mathrm{C}$-reactive protein is a prognostic marker of overall survival. Data for 48 patients with histologically confirmed locally advanced tumors showed significantly increased survival with a higher Jass-Klintrup score $(P=0.037)$. In patients with node-negative disease, 5 -year survival was $49 \%$. However, where there were high-risk pathological characteristics according to the Petersen Index, survival was similar to that for node-positive disease $(P=0.702)$. Our data also showed a significant difference in survival between groups divided according to whether they had a modified Glasgow Prognostic Score of 1 or $2(P=0.031)$.

Conclusion: In order to maintain a reasonable balance between an aggressive approach and so-called meaningless "surgical exorbitance", we should focus on certain histopathological and inflammatory markers that can be identified as additional factors for planning the type and volume of surgical treatment.

Keywords: locally advanced colorectal cancer, C-reactive protein, Jass-Klintrup score, Glasgow Prognostic Score, Petersen Index

\section{Introduction}

Between $5 \%$ and $22 \%$ of colorectal carcinomas become locally advanced tumors. According to the available data, patients with stage IIB tumors, which extend through the intestinal wall and infiltrate the surrounding structures without lymph node metastases (T4N0M0), have worse survival than those with stage IIIA tumors that remain within the intestinal wall but spread to the lymph nodes (T1-2N1/1c-2a). Recent studies confirm that stage T4 is an important and independent predictor of disease-free survival and overall survival. ${ }^{1-3}$ However, there is no consensus on the importance 
of an integrated "multi-team" approach in the preoperative patient evaluation or on the implementation of neoadjuvant and adjuvant chemotherapy and radiotherapy.

Due to the clinical presumption of tumor involvement in other structures and organs, either as primary or recurrent disease, locally advanced colorectal cancer (CRC) is a surgical challenge. Anticipation of the need for extensive surgical resection, often by accomplishment of multivisceral (en bloc) resection, is crucial in the preoperative surgical planning. Long-term postoperative survival is achievable in patients with primary or recurrent locally advanced CRC, but only after complete R0 resection. Neoadjuvant and adjuvant therapy continue to have a role in the present era of biologic chemotherapy. The multimodality treatment approach provides an opportunity for technical realization of resection and improvement of long-term survival.

In order to achieve practical and theoretical clarity regarding the high percentage of patients with $\mathrm{CRC}$, we need a definition of locally advanced disease. Patients with cancer of the colon or rectum may develop a different shape and extent of locally advanced primary or recurrent tumor, that can still be with non-metastatic disease, which can be resected. There are rather vague and not clearly defined criteria for surgical resection at present. Locally advanced CRC is defined by some authors as one being visualized by endorectal ultrasound as a T3/T4N1 tumor. Other authors define it as palpable abdominal tumor mass during physical examination but without the presence of distant metastases. ${ }^{5}$

According to another contemporary definition, an advanced tumor (advanced cancer, advanced malignant disease) is considered as each malignant tumor at initial diagnosis or each recurrent tumor in stage of local development outside of the organ of origin, which is inappropriate for radical resection or presents with distant metastasis. ${ }^{2}$

In our opinion, locally advanced CRC can be assumed when a multidisciplinary team (surgeons, pathologists, radiologists, medical oncologists, gastroenterologists, and specialist in diagnostic images) conclude that a R0 resection cannot be achieved by standard resection of a single organ. Therefore, radical treatment requires a multivisceral resection approach. Locally advanced lesions may range from visible intimately adhered to the surrounding tissue tumor, (marginal, "borderline-resectable" tumor) to such one which penetrates directly in adjacent critical structures (eg, main vessels, duodenum, pancreas, pelvic bones, lateral or anterior abdominal wall, other parts of the colon or small intestine or its mesentery, internal genitalia or organs of the urinary system, nervous plexus). The data indicate that when the primary tumor is in the sigmoid colon or rectum (in $66 \%-89 \%$ of cases), the surrounding structures and organs are affected; the reasons for this are the high incidence of cancer localization in these areas, the mobility of the sigmoid, and the close spatial proximity of the structures in the pelvis. ${ }^{3,6-8}$

Local recurrence emergence after primary treatment of CRC is also a variable concept in terms of its acceptance by the surgeons. Local recurrence after primary treatment of CRC can manifest as a local recurrence with the same histological characteristics or less differentiated tumor at the site of the anastomosis of the previous surgery (which has been resected with subsequent reconstruction/anastomosis) or as a locoregional recurrence in the surrounding tissue of the primary tumor in cases of incomplete R1 resection, inadequate removal of regional lymph vessels or lymph nodes, or extensive perineural invasion that is not covered in the removed specimen, and also iatrogenic embedding of tumor cells in the tumor bed. ${ }^{9}$

Approximately $40 \%$ of patients with resected CRC develop recurrence. Most of them have distant spread of the disease. Locoregional recurrence is much less common, accounting for $10 \%-20 \%$ of recurrent cases. Causes of local recurrence include incomplete resection of transmural or lymphatic spread of the disease, integrity of the tumor, or implantation of tumor cells. Surgery remains the primary treatment. However, it is clear that only $\mathrm{R} 0$ resection can achieve long-term survival.

\section{Inflammation, inflammatory markers, CRP, and locally advanced CRC}

Chronic inflammation is associated with several solid tumor types, including cancers of the esophagus, stomach, liver, pancreas, kidney, and prostate. It is interesting how the levels of inflammatory markers are changing with the volumetric growth of the tumor and progressive involvement of the surrounding structures and organs. The prognostic value of C-reactive protein (CRP) in CRC is of considerable interest.

After reviewing the evidence for an association between CRP and locally advanced and recurrent CRC, we questioned whether such enthusiasm is justified. It has long been accepted that disease progression in patients with CRC is determined not only by local tumor characteristics, but also by systemic immune and inflammatory responses. There is much evidence showing that the local and systemic inflammatory response plays an important role in the progression of a variety of tumor types. ${ }^{10,11}$ 


\section{C-reactive protein}

CRP is a general marker of inflammation. An increased risk of colon cancer has been observed in patients with chronic inflammatory bowel disease, particularly ulcerative colitis. However, inflammatory bowel disease is an etiological factor in only $1 \%-2 \%$ of all cases of colon cancer in the general population. ${ }^{12}$ In laboratory studies, inflammation stimulates cells in the colon to change from adenoma to adenocarcinoma. ${ }^{13}$ Long-term use of aspirin and other nonsteroidal anti-inflammatory drugs is associated with a reduced risk of colorectal adenoma and cancer. ${ }^{14-16}$ However, there is serious debate on the mechanisms via which nonsteroidal anti-inflammatory drugs reduce the risk of $\mathrm{CRC}$, with the evidence suggesting that pathways not related to inflammation may play a significant role. ${ }^{16} \mathrm{CRP}$ is an acute-phase protein and a component of the innate immune response, and increases in response to infection, trauma, burns, tissue necrosis, inflammation, and malignancy. ${ }^{17,18}$ Preoperative CRP levels in patients with advanced CRC are significantly higher than those in patients with early-stage disease..$^{19,20}$ Further, elevated levels of CRP in patients with CRC are related to tumor stage and recurrence as well as reduced survival. ${ }^{21-23}$ In a study by Chung and Chang, CRP levels were increased in one third of 172 patients with CRC before resection; intraoperatively, this was associated with larger tumor size and greater lymph node involvement and metastases to the liver. However, in multivariate analysis, the authors suggested that the CRP level was not an independent predictor of survival. ${ }^{24}$

There are also studies reporting significantly higher levels of CRP and interleukin-6 in patients with CRC than in controls. ${ }^{25-27}$ However, all the data are consistent with the hypothesis that CRP levels are increased after onset of CRC and progressive growth of the tumor, particularly with local advancement. The reverse causality (ie, that CRP is increased as a result of development of (RC), does not necessarily exclude the role of CRP and proinflammatory cytokines in the progression or aggressiveness of the disease. The question as to whether CRP acts as an independent prognostic factor in CRC may not be answered adequately by observational and retrospective studies. The published data show conflicting results and analyses for a causal relationship. They are also limited by significant differences in study design. ${ }^{28,29} \mathrm{~A}$ study reported by Il'yasova et al showed that interleukin-6, tumor necrosis factor, and CRP are inflammation markers associated with risk of cancer of the colon and rectum; moreover, they are significantly elevated in cases of locally advanced CRC. High levels of CRP are also associated with morbidity from cancer of the colon and rectum. ${ }^{30}$
The interpretation of CRP levels in patients with CRC rouse a lot of interest. However, while measurement of CRP is feasible and convenient, further evaluation in carefully designed studies is needed to determine whether CRP has a role as a diagnostic and prognostic index in CRC.

\section{Jass-Klintrup score}

There is strong evidence that the pronounced lymphocytic infiltration observed on standard hematoxylin-eosin staining in and around the tumor in localized CRC cases is associated with improved survival. ${ }^{31}$ Klintrup al subjectively measured inflammatory infiltration of tumor tissue by including all cell types of white cells and classifying them as low-grade or high-grade, and concluded that a high degree of inflammatory infiltration is associated with improved survival in patients who undergo potentially curative resection of lymph node-negative CRC. ${ }^{31}$ The Klintrup tumor staging system includes four possible scores:

- 0 , no increase in the number of inflammatory cells in the deep layers of the invasive tumor edge

- 1 , indicates an average or piecemeal increase in inflammatory cells

- 2 , indicates a pronounced inflammatory reaction and formation of areas of destruction (like "islands" of destructed tumor cells) in the deep layers of the invasive tumor edge

- 3 , denotes abundant inflammatory infiltration in the invasive tumor edge by frequent "islands" of destructed tumor cells (as piecemeal-tumor necroses).

The most commonly used version of this system is a modified score, ie, low (score $0-1$ ) and high (score 2-3). ${ }^{32}$ Jass et al proposes a score system consider "the peritumoral lymphocytic infiltration" as a part of the "stromal reaction" in the tumor invasive edge.

Specific features included in the score are: presence or absence of connective tissue strands or layers in the deep layers of the tumor invasive edge; marked infiltration of neutrophils, macrophages, eosinophils, lymphocytes, and plasma cells between the glandular structures of the tumor tissue; and, most importantly, the presence or absence of connective tissue stroma. Tumors are staged using a 2-point scale, based on whether peritumoral infiltration is present or not. ${ }^{33,34}$

The prognostic Jass score is defined by:

- T category - limited tumor growth in the intestinal wall (0 points); tumor growth outside the intestinal wall, ie, T4 (one point)

- Number of metastatic lymph nodes - 0 (0 points), 1-4 (one point), $>4$ (two points) 
- Invasiveness of the tumor edge - expansive form (0 points), infiltrative form (one point)

- Peritumoral lymphocytic infiltration - expressed (0 points), not expressed/missing (one point)

- Score group - I (0-1), II (2), III (3), and IV (4-5); low degree (group I-II, score 0-2); high degree (group III-IV, score 3-5).

\section{Petersen Index}

Additional clinically useful, pathologically defined criteria that provide independent prognostic information after resection of node-negative CRC were identified by Shepherd et al. ${ }^{35}$ These are peritoneal involvement, venous invasion (submucosal and extramural), involving resection margins, and perforation of the tumor. The so-called Petersen Index (PI) combines these four factors in one scoring system. The outlook for the patient worsens as the number of these characteristics present increases. Thus, in the group with nodenegative disease, which accounts for $40 \%-50 \%$ of surgical patients with resected CRC, the reported 5-year survival is $75 \%-90 \% .{ }^{36}$ However, when there are high-risk pathological features, survival is similar to that in node-positive CRC. ${ }^{37}$ The PI identifies patients at high risk of tumor recurrence who may benefit from adjuvant treatment and confirms Dukes stage B and C (with positive lymph nodes) CRC. ${ }^{38}$

\section{Determination of PI on histological examination}

The PI in surgical patients with locally advanced CRC (T4 tumors, stage IIB, stage IIIB, stage IIIC) was defined by analyzing the histological results jointly with the Department of Pathology. This index is calculated by four histopathological features:

- Peritoneal involvement (+1 point)

- Tumor invasion of resection margins, transverse or tcircumferential (+1 point)

- Venous invasion, V1 (+1 point)

- Tumor perforation (+2 points).

Patients with scores of $0-1$ are considered low-risk, while patients with a total score $\geq 2$ are considered high-risk for local recurrence and, accordingly, aggressive adjuvant therapy is recommended.

\section{Glasgow Prognostic Score}

There is considerable evidence that manifestation of a systemic inflammatory response, indicated by the Glasgow Prognostic Score (GPS), based on increased CRP levels and hypoalbuminemia, is independently associated with a worse outcome in patients with CRC. ${ }^{39}$ The GPS allocates a score of 2 for patients with increased CRP $(>10 \mathrm{mg} / \mathrm{L})$ and hypoalbuminemia $(<35 \mathrm{mg} / \mathrm{L})$, a score of 1 for patients with only one of these parameters, and a score of 0 for patients with neither of these parameters. This system has now been modified based on evidence that hypoalbuminemia in patients with locally advanced CRC and no increase in the CRP is not significantly associated with cancer-specific survival. Thus, patients with elevated CRP are included in the modified GPS (mGPS) score, with a score of 1 or 2 depending on the presence or absence of hypoalbuminemia. ${ }^{40}$

Roxburgh et al were the first to demonstrate a relationship between the preoperative systemic inflammatory response (according to GPS criteria), peritumoral inflammatory infiltration (according to Jass-Klintrup criteria), and cancer-specific survival in patients who undergo potentially curative resection for CRC. Low peritumoral inflammation (more a Klintrup criterion, associated with a higher Dukes stage and increased circulating leukocytes (in total) and neutrophils (in particular)) are independently associated with cancer-specific survival. On the other hand, the systemic inflammatory response (mGPS) is not associated with either Dukes stage or peritumoral inflammation, but with increased levels of circulating leukocytes, in particular increased neutrophils. ${ }^{41}$ In terms of indications for surgical treatment or alternative treatment regimes, as well as specific follow-up, the clinical use of all these criteria, along with traditional risk factors, would help to stratify patients with locally advanced CRC into specific groups. ${ }^{42}$

The groups of mGPS identified by these markers are defining the systemic inflammatory responses as follows:

- Score $2-$ CRP levels $(>10 \mathrm{mg} / \mathrm{L})+$ hypoalbuminemia $(<35 \mathrm{mg} / \mathrm{L})$

- Score 1 - CRP levels ( $>10 \mathrm{mg} / \mathrm{L})+$ normoalbuminemia $(>35 \mathrm{mg} / \mathrm{L})$

- Score 0 - CRP levels $(<10 \mathrm{mg} / \mathrm{L})+$ serum albumin $(>35 \mathrm{mg} / \mathrm{L})$.

\section{Materials and methods}

We investigated patients with confirmed CRC who were treated in the surgical clinic at the University Hospital Alexandrovska between 2002 and 2012. Data collection was based on documented evidence from the medical history, operative report, operative registry, data from the Clinical Center of Pathology, and oncology committee protocols.

We analyzed the histological features of preoperative biopsies and histological examination of operative specimens that were removed during radical multivisceral resection. 
We collected prospective data on serum CRP levels and Jass-Klintrup, PI, and GPS scores in patients with locally advanced CRC.

The statistical analysis was performed using IBM Statistical Package for the Social Sciences software. The total number of patients was 1,105 , which ensured representation of all patient groups that are differentiated by certain signs and good reliability of all results. This study had a retrospective cohort design. In accordance with evidence-based medicine, the study had a score of 4 on the 5-point scale for level of evidence and grade of recommendation. All ethical requirements in the science research have been implemented. The Review Board of the University Hospital Alexandrovska Sofia gave consent for the use of the retrospective data of the patients from years 2002-2012.

\section{Results}

During the study period, 1,105 patients $(57.73 \%$ male and $42.27 \%$ female) were identified as having histologically confirmed CRC, of whom 327 (29.6\%) had locally advanced CRC. In $54.5 \%$ of cases, the tumor was localized in various sites of the colon and $45.5 \%$ of cases were in the rectum. Most of the patients was aged between 61 and 80 years. In total, 108 combined multivisceral resections were performed ( 79 for primary tumors and 29 for recurrent tumors); in 17 cases (eight involving recurrent tumors), only R1 could be achieved, ie, radical surgery was impossible. We found preoperative and intraoperative generalization of the cancer process, ie, bilobar unresectable multiple liver metastases and/or diffuse carcinosis in the peritoneum, and palliative surgery was performed in 219 of cases. The palliative procedures used included resections (with or without restoration of the intestinal continuity), bypasses, anastomoses, and simple interruptions of the passage, including cryodestruction. Nineteen patients (17.6\%) with locally advanced disease developed local recurrence 2-5 years after the initial resection; eleven of these patients underwent "radical" repeat en bloc multivisceral resection. In eight cases, a pR0 result was achieved. In the prospective portion of this study, we investigated already known inflammatory and tumor markers.

\section{Determination of CRP}

We investigated CRP in 182 patients with suspected locally advanced primary or recurrent tumors. The frequency of distribution of the different CRP levels is shown in Figure 1. Figure 2 shows the distribution of patients $(n=182)$ according to their CRP level. When we investigated the distribution of CRP levels between groups with early, locally advanced,

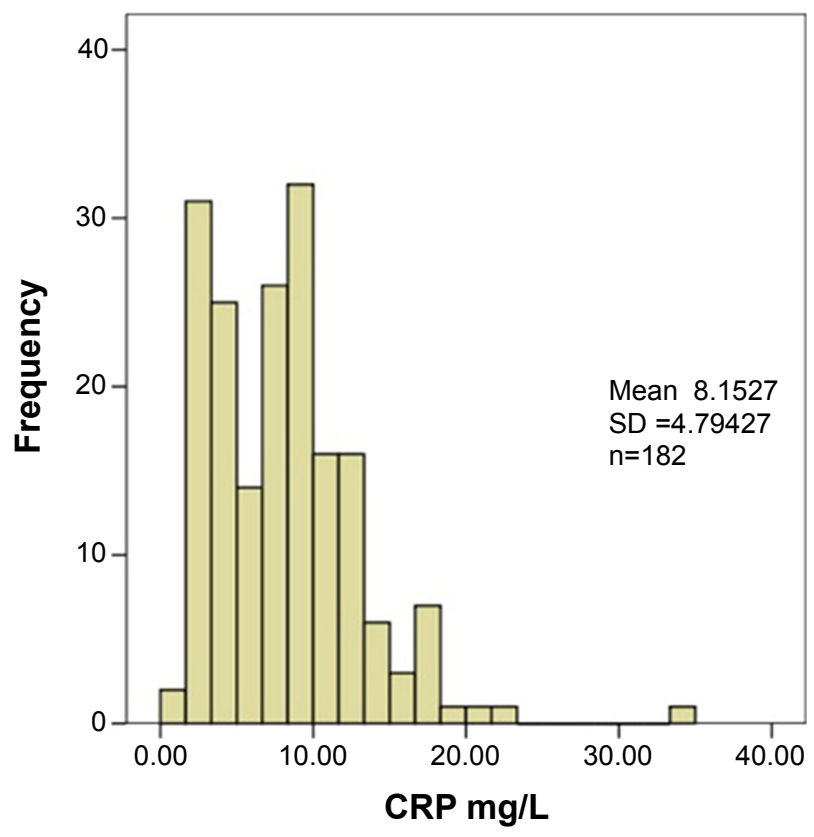

Figure I Frequency of distribution of CRP values (tested preoperatively) in the study population.

Abbreviations: CRP, C-reactive protein; SD, standard deviation.

or recurrent CRC (see Figure 3), we found that CRP was increased in most of the cases of locally advanced cancer.

We then investigated overall survival according to CRP levels $(0-5 \mathrm{mg} / \mathrm{L}, 5-10 \mathrm{mg} / \mathrm{L}$, and $>10 \mathrm{mg} / \mathrm{L})$. The Kaplan-Meier survival curve is shown in Figure 4. In the group with CRP $<5 \mathrm{mg} / \mathrm{L}$, median survival was 36.903 (32.836 -40.970) months; in the group with CRP 5-10 mg/L, median survival was 21.962 (19.511-24.413) months; and in the group with CRP $>10 \mathrm{mg} / \mathrm{L}$, median survival was 11.600 (8.887-13.313) months.

The log rank test showed a statistically significant difference in survival between the groups with $\mathrm{CRP}<5 \mathrm{mg} / \mathrm{L}$ and those with CRP 5-10 mg/L $(P=0.003)$, and between the

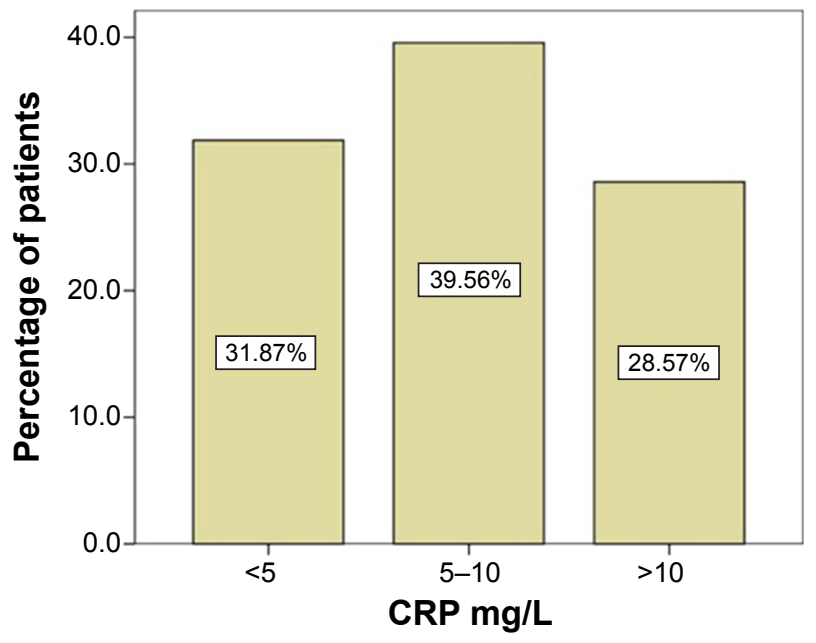

Figure 2 Distribution of patients per their CRP-serum level. Abbreviation: CRP, C-reactive protein. 


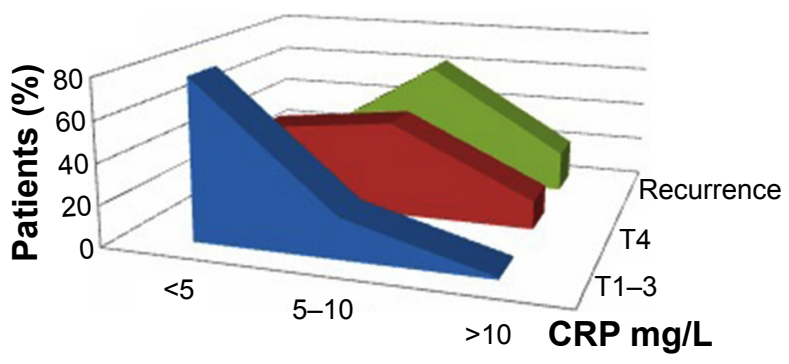

\begin{tabular}{|l|l|l|l|}
\cline { 2 - 4 } \multicolumn{1}{c|}{} & $\mathbf{< 5} \mathbf{~ m g / L}$ & $\mathbf{5 - 1 0} \mathbf{~ m g} / \mathbf{L}$ & $>\mathbf{1 0} \mathbf{~ m g} / \mathbf{L}$ \\
\hline$\square$ T1-3 & $79 \%$ & $20 \%$ & $1 \%$ \\
\hline$\square$ T4 & $39 \%$ & $47 \%$ & $14 \%$ \\
\hline Recurrence & $18 \%$ & $62 \%$ & $20 \%$ \\
\hline
\end{tabular}

Figure 3 Distribution of CRP according to PT category. Abbreviations: CRP, C-reactive protein; $\mathrm{PT}$, primary tumor.

groups with $\mathrm{CRP}<5 \mathrm{mg} / \mathrm{L}$ and $\mathrm{CRP}>10 \mathrm{mg} / \mathrm{L}(P=0.0024)$. There was also a statistically significant difference between the groups with CRP $5-10 \mathrm{mg} / \mathrm{L}$ and CRP $>10 \mathrm{mg} / \mathrm{L}$ $(P=0.004)$. The results are shown in Table 1 , and confirm $\mathrm{CRP}$ as a prognostic factor for overall survival.

\section{Jass-Klintrup score}

The definition of histological and inflammatory markers of locally advanced CRC in the current study was based on the assumption that the presence of localized CRC increases lymphocytic infiltration in and around the tumor (observed by standard hematoxylin-eosin staining) and is associated with improved survival.

With the assistance of the Department of Clinical Pathology and histopathological examination of biopsies and resected specimens, we investigated the local inflammatory response in the tumor tissue. We also evaluated lymphocytic tumor infiltration and determined low-grade and high-grade JK scores in terms of survival rate after potentially curative resection in patients with locally advanced tumors. So-called "peritumoral lymphocytic infiltration" was defined, which reflected the stromal response at the invasive edge of the tumor.

Data from the 48 patients with histologically confirmed locally advanced tumors showed significantly increased

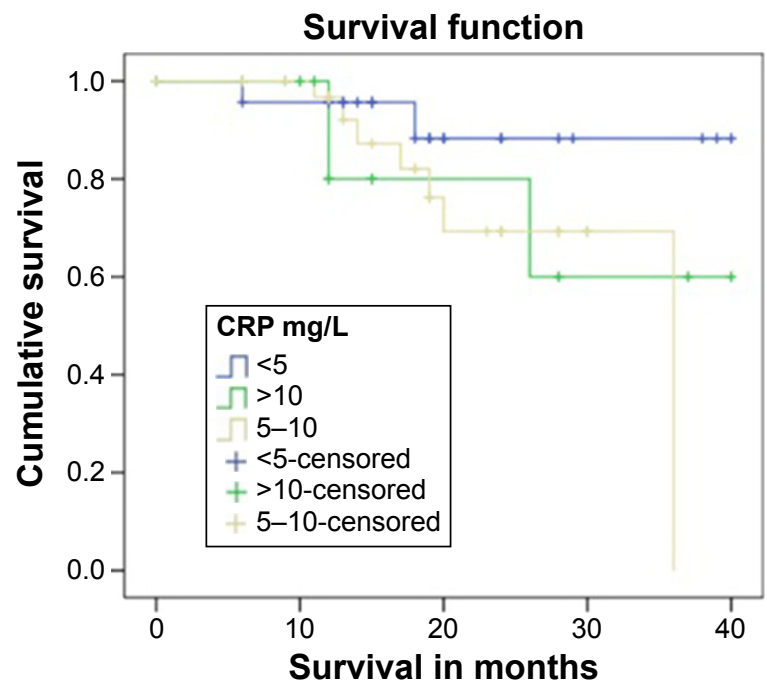

Figure 4 Survival in the study population according to CRP level as a risk factor for survival.

Abbreviation: CRP, C-reactive protein.

survival in those with higher scores indicating a more pronounced infiltration of the tumor edge in the inflammatory cells of the local immune response $(\mathrm{P}=0.0370)$, as shown in Figure 6.

\section{Petersen Index}

Additional clinically relevant prognostic criteria were identified by postoperative histological examination of the removed specimens. These specimens provided important information regarding resected node-negative locally advanced CRCs, including peritoneal involvement $(\mathrm{P}+, \mathrm{P}-)$, venous invasion (submucosal, extramural; V0/V1), involvement of resection margins (R0/R1), and tumor perforation. We added perineural invasion $(\mathrm{PN}+, \mathrm{PN}-)$, thereby modifying the PI score and combining these five factors in a scoring system as follows: $0-5$ ( 0 , absence of any positive factors; $1-4$, presence of 1-4 positive factors, respectively; and 5, presence of all factors). Thus, in the group with node-negative disease, which accounted for $44 \%$ of our patients with cT 4 carcinoma, 5-year survival was $49 \%$. In patients with a high PI score, survival was similar to that for node-positive disease $(P=0.702)$. The

Table I Log rank test for survival according to CRP level

\begin{tabular}{|c|c|c|c|c|c|c|c|}
\hline \multicolumn{8}{|l|}{ Pairwise comparisons } \\
\hline & \multirow{2}{*}{$\begin{array}{l}\text { CRP level } \\
(\mathrm{mg} / \mathrm{L})\end{array}$} & \multicolumn{2}{|c|}{$<5 \mathrm{mg} / \mathrm{L}$} & \multicolumn{2}{|c|}{$5-10 \mathrm{mg} / \mathrm{L}$} & \multicolumn{2}{|c|}{$>10 \mathrm{mg} / \mathrm{L}$} \\
\hline & & $\chi^{2}$ & Significance & $\chi^{2}$ & Significance & $\chi^{2}$ & Significance \\
\hline \multirow[t]{3}{*}{ Log rank (Mantel-Cox) } & $<5$ & & & 2.420 & 0.0031 & 1.550 & 0.002 \\
\hline & $5-10$ & 2.420 & 0.003 & & & 0.000 & 0.004 \\
\hline & $>10$ & 1.550 & 0.002 & 0.000 & 0.004 & & \\
\hline
\end{tabular}

Abbreviation: CRP, C-reactive protein. 


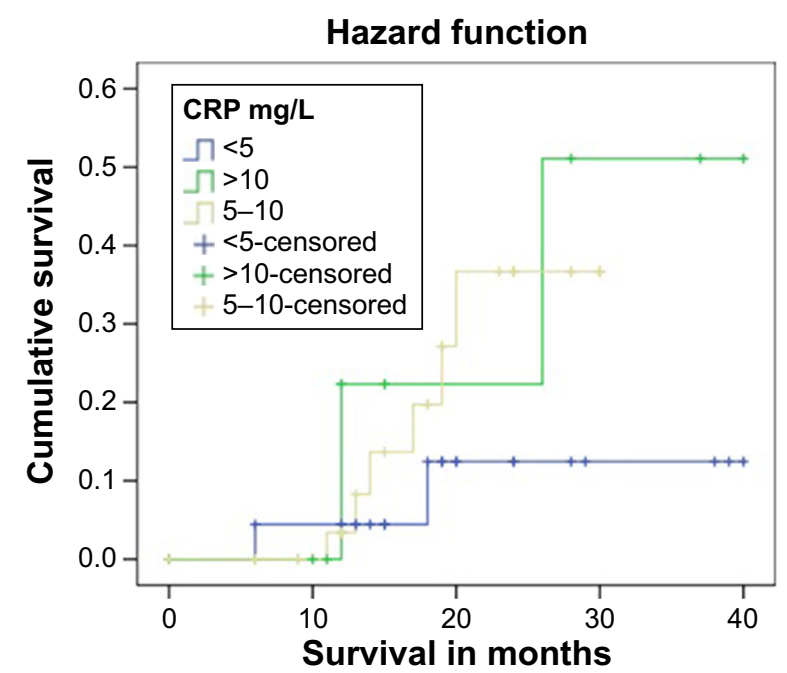

Figure 5 Higher CRP level as a risk factor for survival rate. Abbreviation: CRP, C-reactive protein.

PI identified patients at high risk of tumor recurrence who would benefit from aggressive adjuvant treatment and had cT4 tumors (Dukes' stage B or C, with a positive node) as shown in Figure 7.

\section{Modified Glasgow Prognostic Score}

The systemic immune inflammatory response was determined by the mGPS, which takes into account the number of patients with preoperatively defined serum CRP $(>10 \mathrm{mg} / \mathrm{L})$ and hypoalbuminemia $(<35 \mathrm{mg} / \mathrm{L})$ on the basis of evidence that hypoalbuminemia in patients with locally advanced CRC without an increase in CRP was not significantly associated with cancer-specific survival. Thus, patients with preoperative CRP elevation were allocated an mGPS score of 1 or 2, depending on the presence or absence of preoperative hypoalbuminemia.

The data showed a statistically significant difference in survival between the group with a score of 1 and the group with a score of 2 ( $P=0.0311$, Figure 8$)$. The importance of the mGPS as a prognostic indicator was reflected in the fact that it was not connected with either Dukes stage or peritumoral

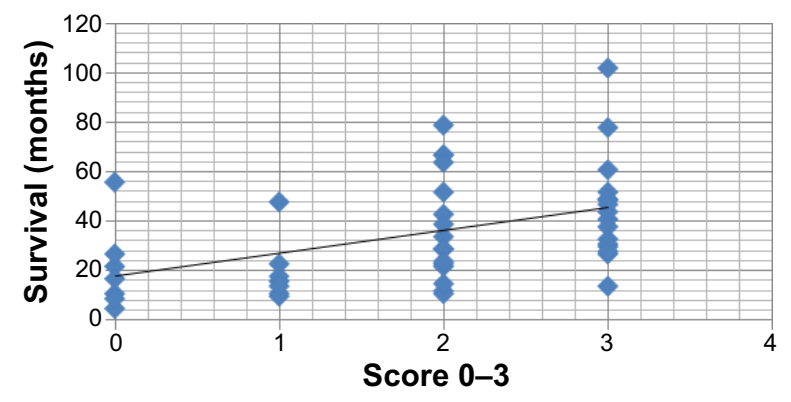

Figure 6 Jass-Klintrup distribution in the group with pT4 locally advanced tumors.
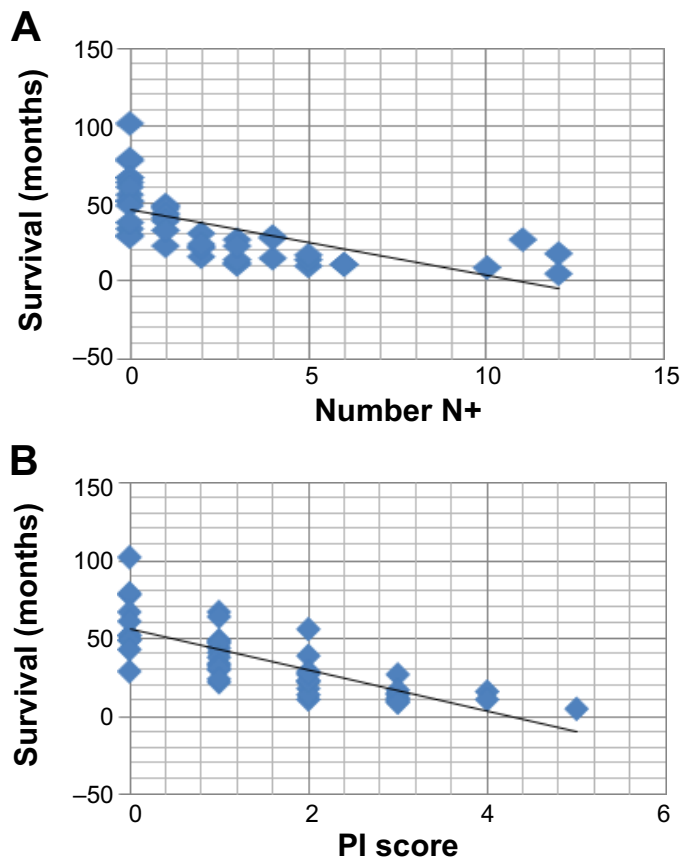

Figure 7 Comparison of the modified $\mathrm{PI}$ in the study population $(\mathbf{A})$ with $\mathrm{PT} 4 \mathrm{~N} 0 / \mathrm{I}$ (one positive lymph node) and survival depending on number of metastatic $(\mathrm{N}+)$ lymph nodes (B)

Abbreviation: PI, Petersen index.

inflammation, but was associated with increased levels of circulating leukocytes, in particular increased neutrophils, in addition to decreased lymphocytes. The mGPS showed a significant correlation with poorer cancer-related survival. The basis for this is that systemic inflammatory markers such as CRP play a central role in the relationship between the tumor and its host. Better preoperative evaluation of patients and separation into groups in terms of additional indications for aggressive behavior or palliative surgical procedures is needed.

\section{Discussion}

Many world-famous surgical centers recommend the "adequate aggressive behavior" for locally advanced primary and

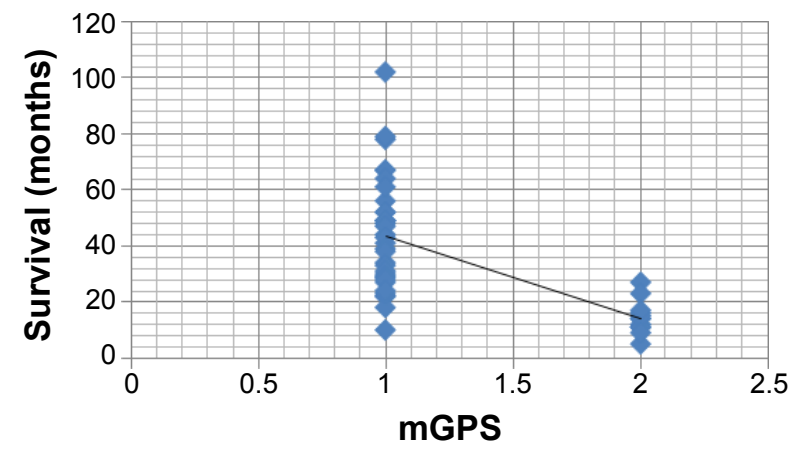

Figure 8 Relationship between mGPS and survival in patients with pT4 disease. Abbreviation: mGPS, modified Glasgow Prognostic Score. 
recurrent colorectal carcinomas. ${ }^{14}$ This inevitably leads to a need for individualized and comprehensive assessment of each case, using all diagnostic methods available for preoperative staging, as well as a broad discussion of the results by a multidisciplinary team before embarking on surgery. Our vision largely overlaps with the opinion of most of the published authors with regard to the circumstances that determine decision-making regarding the possible resectability of locally advanced CRC.

We should also focus on the importance of certain histopathological and inflammatory markers identified in the preoperative process with regard to the prognosis. This markers are additional factors in planning the type and volume of surgical treatment. Our study data for Jass-Klintrup scoring of histologically confirmed locally advanced tumors showed significantly increased survival in patients with higher scores. That indicates more pronounced infiltration of the tumor edge by inflammatory cells involved in the local immune response $(P=0.0370)$, and underscores the importance of obtaining a sufficient number and volume of macrobiopsies taken from the tumor tissue closest to the tumor edge in the preoperative period and defining the relevant Jass-Klintrup score. Performing the multivisceral resection would be helpful for making the decision on which aggressive approach may be used for the treatment of locally advanced tumors. Determination of preoperative CRP levels as part of the mGPS in this study showed a significant difference in survival between the group with a score of 1 and the group with a score of 2 $(P=0.0311)$. A higher score was shown to have a significant relationship with shorter cancer-related survival. Preoperative assessment of the mGSP would be helpful in separating patients with locally advanced CRC into specific groups, in terms of employing a more aggressive surgical approach. The modified PI used in the present study for patients with histologically confirmed pT4 disease showed that in the group with node-negative disease and a low PI score, observed in $49 \%$ of the cases, 5 -year survival was significantly different from that in the group with high-grade pathological characteristics according to the PI, in which survival was similar to that of patients with node-positive disease $(P=0.702)$. The PI is useful for selecting patients with a high risk of tumor recurrence. Those patients are likely to benefit from complete multivisceral resection followed by aggressive adjuvant therapy. Validation of a standardized strategy for management of patients with advanced (locally or generalized) CRC and definition of clear criteria for resectability of the primary or the recurrent tumor are imperative.

\section{Disclosure}

The authors report no conflicts of interest in this work.

\section{References}

1. Guillem JG, Chessin DB, Cohen AM, et al. Long-term oncologic outcome following preoperative combined modality therapy and total mesorectal excision of locally advanced rectal cancer. Ann Surg. 2005; 241:829-836.

2. Young A, Rea A. Treatment of advanced disease. $B M J$. 2000;321: 1278-1281.

3. Oh SY, Kim YB, Paek OJ, Suh KW. Contiguous invasion per se does not affect prognosis in colon cancer. J Surg Oncol. 2009;99:71-74.

4. Willett CG, Rodriguez-Bigas MA, Ryan DP. Treatment of locally advanced unresectable or recurrent rectal cancer. Available from: http://www.uptodate.com/contents/treatment-of-locally-advancedunresectable-or-recurrent-rectal-adenocarcinoma. Accessed May 10, 2015.

5. American Joint Committee on Cancer. AJCC Cancer Staging Manual. 6th ed. New York, NY, USA: Springer; 2002.

6. Curley SA, Evans DB, Ames FC. Resection for cure of carcinoma of the colon directly invading the duodenum or pancreatic head. $J$ Am Coll Surg. 1994;179:587-592.

7. Polk HC Jr. Extended resection for selected adenocarcinomas of the large bowel. Ann Surg. 1972;175:892-899.

8. Pittam MR, Thornton H, Ellis H. Survival after extended resection for locally advanced carcinomas of the colon and rectum. Ann R Coll Surg Engl. 1984;66:81-84.

9. de Wilt JH, Vermaas M, Ferenschild F, Verhoef C. Management of locally advanced primary and recurrent rectal cancer. Clin Colon Rectal Surg. 2007;20:255-263.

10. Mantovani A, Romero P, Palucka AK, et al. Tumor immunity: effector response to tumor and role of the microenvironment. Lancet. 2008;371:771-783.

11. Vakilla J, Lotze MT. Inflammation and necrosis promote tumor growth. Nat Cancer Rev. 2004;4:641-648.

12. Munkholm P. Review article: the incidence and prevalence of colorectal cancer in inflammatory bowel disease. Aliment Pharmacol Ther. 2003;18 Suppl 2:1-5.

13. Okada F, Kawaguchi T, Habelhah H, et al. Conversion of human colonic adenoma cells to adenocarcinoma cells through inflammation in nude mice. Lab Invest. 2000;80:1617-1628.

14. Baron JA, Cole BF, Sandler RS, et al. A randomized trial of aspirin to prevent colorectal adenomas. N Engl J Med. 2003;348:891-899.

15. Baron JA. Epidemiology of non-steroidal anti-inflammatory drugs and cancer. Prog Exp Tumor Res. 2003;37:1-24.

16. Huls G, Koornstra JJ, Kleibeuker JH. Non-steroidal anti-inflammatory drugs and molecular carcinogenesis of colorectal carcinomas. Lancet. 2003;362:230-232.

17. Coussens LM, Werb Z. Inflammation and cancer. Nature. 2002;420: $860-867$.

18. Gabay C, Kushner I. Acute-phase proteins and other systemic responses to inflammation. N Engl J Med. 1999;340:448-454.

19. Durdey P, Williams NS, Brown DA. Serum carcinoembryonic antigen and acute phase reactant proteins in the pre-operative detection of fixation of colorectal tumours. Br J Surg. 1984;71:81-84.

20. Mercer DW, Talamo TS. Multiple markers of malignancy in sera of patients with colorectal carcinoma: preliminary clinical studies. Clin Chem. 1985;31:1824-1828.

21. McMillan DC, Wotherspoon HA, Fearon KC, McArdle CS. A prospective study of tumor recurrence and acute-phase response after apparently curative colorectal cancer. Am J Surg. 1995;170:319-322.

22. Nozoe T, Matsumata T, Kitamura M, Sugimachi K. Significance of preoperative elevation of serum C-reactive protein as an indicator for prognosis in colorectal cancer. Am J Surg. 1998;176:335-338. 
23. Wigmore SJ, McMahon AJ, Sturgeon CM, Fearon KC. Acute-phase protein response, survival and tumour recurrence in patients with colorectal cancer. Br J Surg. 2001;88:255-260.

24. Chung YC, Chang YF. Serum C-reactive protein correlates with survival in colorectal cancer patients but is not an independent prognostic indicator. Eur J Gastroenterol Hepatol. 2003;15:369-373.

25. Chung YC, Chang YF. Serum interleukin-6 levels reflect the disease status of colorectal cancer. J Surg Oncol. 2003;83:222-226.

26. Kinoshita T, Ito H, Miki C. Serum interleukin-6 level reflects the tumor proliferative activity in patients with colorectal carcinoma. Cancer. 1999 ; 85:25-26.

27. Yuceyar S, Ertuk S, Dirican A, Cengiz A, Saner H. The role of acutephase reactant proteins, carcinoembryonic antigen and CA 19-9 as a marker in the preoperative staging of colorectal cancer: a prospective clinical study. Int Surg. 1996;81:136-139.

28. Erlinger TP, Platz EA, Rifai N, Helzlsouer KJ. C-reactive protein and the risk of incident colorectal cancer. JAMA. 2004;29:585-590.

29. Ko WF, Helzlsouer KJ, Comstock GW. Serum albumin, bilirubin, and uric acid and the anatomic site-specific incidence of colon cancer. J Natl Cancer Inst. 1994;86:1874-1875.

30. Il'yasova D, Colbert LH, Harris TB, et al. Circulating levels of inflammatory markers and cancer risk in the health aging and body composition cohort. Cancer Epidemiol Biomarkers Prev. 2005;14:2413-2418.

31. Galon J, Costes A, Sanchez-Cabo F, et al. Type, density, and location of immune cells within human colorectal tumors predict clinical outcome. Science. 2006;313:1960-1964.

32. Klintrup K, Makinen JM, Kauppila S, et al. Inflammation and prognosis in colorectal cancer. Eur J Cancer. 2005;41:2645-2654.

33. Jass JR, Ajikoka Y, Allen JP, et al. Assessment of invasive growth pattern and lymphocytic infiltration in colorectal cancer. Histopathology. 1996;28:543-548.
34. Jass JR, Love SB, Northover JM. A new prognostic classification of rectal cancer. Lancet. 1987;1:1303-1306.

35. Shephard EG, Anderson R, Rosen O, et al. Peptides generated from $\mathrm{C}$-reactive protein by a neutrophil membrane protease. Amino acid sequence and effects of peptides on neutrophil oxidative metabolism and chemotaxis. J Immunol. 1990;145(5):1469-1476.

36. McArdle CS, McKee RF, Findlay IG, Wotherspoon H, Hole DJ. Improvement insurvival following surgery for colorectal cancer. $\mathrm{Br} J$ Surg. 2005;92:1008-1013.

37. Figueredo A, Coombes ME, Mukherjee S. Adjuvant therapy for completely resected Stage II colon cancer. Cochrane Database Syst Rev. 2008;3:CD005390.

38. Morris EJ, Maughan NJ, Forman D, Quirke P. Who to treat with adjuvant therapy in Dukes B/stage II colorectal cancer? The need for high quality pathology. Gut. 2007;56:1419-1425.

39. Ishizuka M, Nagata $H$, Takagi $K$, et al. Inflammation-based prognostic score is a novel predictor of postoperative outcome in patients with colorectal cancer. Ann Surg. 2007;246:1047-1051.

40. Forrest LM, McMillan DC, McArdle CS, et al. Evaluation of cumulative prognostic scores based on the systemic inflammatory response in patient inoperable non-small-cell lung cancer. Br J Cancer. 2003;89: 1028-1030.

41. Roxburgh CS, Salmond JM, Horgan PG, Oien KA, McMillan DC. Comparison of the prognostic value of inflammation-based pathologic and biochemical criteria in patients undergoing potentially curative resection for colorectal cancer. Ann Surg. 2009;249:788-793.

42. Crozier JE, Mckee RF, McArdle CS, et al. The presence of a systemic inflammatory response predicts poorer survival in patients receiving adjuvant 5-FU chemotherapy following potentially curative resection for colorectal cancer. Br J Cancer. 2006;94:1833-1836.
OncoTargets and Therapy

\section{Publish your work in this journal}

OncoTargets and Therapy is an international, peer-reviewed, open access journal focusing on the pathological basis of all cancers, potential targets for therapy and treatment protocols employed to improve the management of cancer patients. The journal also focuses on the impact of management programs and new therapeutic agents and protocols on

\section{Dovepress}

patient perspectives such as quality of life, adherence and satisfaction. The manuscript management system is completely online and includes a very quick and fair peer-review system, which is all easy to use. Visit http://www.dovepress.com/testimonials.php to read real quotes from published authors. 